\title{
Adjuvant Chemotherapy Treatment after Radical Cystectomy in Patients with Muscle Invasive Bladder Cancer Hassan Khaled Hamdy ${ }^{1}$, Mohsen Salah El-Din Zekry ${ }^{1}$, Sabri Mahmoud Khaled ${ }^{2}$ and Sherif Mohammed Mustafa Azzam ${ }^{1 *}$ \\ ${ }^{1}$ Clinical Oncology and Nuclear Medicine, and ${ }^{2}$ Urology and Andrology Departments, Faculty of Medicine, Al-Azhar University, Cairo, Egypt \\ *Corresponding Author: Sherif Mohammed Mustafa Azzam, E-mail: sherifazzam24@yahoo.com
}

\begin{abstract}
Background: about $25 \%$ of patients newly diagnosed with bladder cancer have muscle-invasive bladder cancer (MIBC). Patients with MIBC have a worse prognosis than those with non-MIBC. Radical cystectomy with pelvic lymphadenectomy has been shown to be effective against MIBC. The pathologic stage of the primary tumor and regional lymph nodes status has been shown to be the most accurate predictors of disease recurrence after radical cystectomy.

Aim of the Work: to evaluate the toxicity profile related to the adjuvant chemotherapy cisplatin, gemcitabine when added to radical cystectomy as primary treatment, and to estimate disease free survival (DFS) and overall survival (OS).

Patients and Methods: during the period between December 2013 and October, 2017, a total number of 42 patients were included in this study at Clinical Oncology and Nuclear Medicine Department, AlHussein University Hospital with a provisional diagnosis of invasive type bladder cancer. The cutoff date for the analysis of overall survival was $31^{\text {st }}$ April, 2018 corresponding to 6 months of follow-up for the last patient enrolled in the study. All patients were subjected to radical cystectomy and pelvic lymphadenectomy and received four cycles of adjuvant chemotherapy cisplatin $70 \mathrm{mg} / \mathrm{m}^{2}$ D1, gemcitabine $1000 \mathrm{mg} / \mathrm{m}^{2} \mathrm{D} 1,8$, every three weeks.

Results: the most common grade 3 and 4 adverse events of hematological and non-hematological toxicities recorded during adjuvant chemotherapy were neutropenia (18.8\%), grade 3 anemia $(9.5 \%)$, grade 3 thrombocytopenia (2.3\%), grade 3 nausea (28.5\%), grade 3 and 4 vomiting (9.4\%), grade 3 diarrhea (9.4\%) while grade 3 renal toxicities observed in two patients $(4.7 \%)$. As regard the survival analysis, the median disease-free survival (DFS) rate was not reached due to a relatively short follow up period and DFS was $82.9 \%$ at 1 year, $74 \%$ at 2 years, and $70.1 \%$ at 3 years. Concerning overall survival analysis, the median overall survival in our study was not reached due to a relatively short follow up period. Overall Survival rate at 1 year was $90.4 \%$; at 2 years was $77.3 \%$ and $73.4 \%$ at 3 years.

Conclusion: for patients with bladder cancer who were not treated with neoadjuvant chemotherapy, we suggest not routinely administering chemotherapy following cystectomy. However, for patients with high-risk (T3 or higher, pathologic node involvement) urothelial carcinomas who are candidates for cisplatin -based combination chemotherapy and are willing to accept the risk for treatment-related toxicities in the absence of high level of evidence, adjuvant chemotherapy is a reasonable option. If administered, we prefer to use a cisplatin-based combination.
\end{abstract}

Keywords: Chemotherapy - Radical Cystectomy - Muscle Invasive Bladder

\section{INTRODUCTION}

Radical cystectomy is the standard treatment for patients with muscle invasive bladder cancer. Five-year survival for patients with pT3-pT4a pN0 M0 bladder cancer after radical cystectomy is $35 \%-40 \%$. In $\mathrm{pN}+$ patients, five year survival is no more than $10 \%$ (1).

Surgical approaches, including en bloc cystectomy, bilateral pelvic iliac lymph node dissection, and various forms of lower urinary tract reconstruction, have been developed to enhance survival in patients with MIBC. Improvements in medical, surgical, and anesthetic methods have reduced the morbidity and mortality associated with surgery. Radical cystectomy provides an accurate evaluation of both the primary bladder tumor and the regional lymph nodes, allowing for adjuvant treatment strategies based on clear pathologic rather than clinical staging ${ }^{(2)}$.

Invasive bladder cancer is generally a lethal disease requiring aggressive therapy, with fewer than $15 \%$ of untreated patients surviving to $2 \mathrm{yr}$ after diagnosis. The optimal goals of treatment for any invasive bladder cancer include long-term survival, prevention 
of pelvic recurrence or development of metastatic bladder cancer, and an excellent quality of life ${ }^{(\mathbf{1})}$.

Meta-analysis of nine RCTs (five previously analyzed, one updated, and three new), which included 945 patients, was performed in $2013^{(3)}$. It showed $\mathbf{2 3 \%}$ relative decrease in the risk of death with AC compared with controls and $\mathbf{3 4 \%}$ relative decrease in the risk of recurrence. Although it was thought that this updated meta-analysis offered further evidence of OS and DFS benefits, there were some limitations and it is still controversial. First, individual patient's data (IPD) for this meta-analysis was not available. Second, the Italian ${ }^{(4)}$ and Spanish trials ${ }^{(5)}$ had completely opposite results.

Generally, adjuvant chemotherapy is recommended for patients with high-risk features such as T3 or T4 disease and/or lymph node involvement, who have not been treated with neoadjuvant chemotherapy. Routine use of adjuvant chemotherapy in patients with MIBC without such high-risk features is controversial due to the lack of evidence of clear benefit in this group. There are limited data on the management of patients who are not candidates for cisplatinbased adjuvant chemotherapy ${ }^{\left({ }^{(}\right)}$.

The primary aim of this work was to evaluate the toxicity profile related to the adjuvant chemotherapy cisplatin, gemcitabine when added to radical cystectomy as primary treatment. A secondary endpoint was to estimate disease free survival (DFS) and overall survival (OS). A tertiary objective was to describe the associations between pathologic features and lymph node density to clinical outcomes.

\section{PATIENTS AND METHODS}

This study included a total of 42 patients with a provisional diagnosis of invasive type bladder cancer attending at Clinical Oncology and Nuclear Medicine Department, Al-Hussein University Hospitals. Approval of the ethical committee and a written informed consent from all the subjects were obtained. This study was conducted between December 2013 and October, 2017.

The cutoff date for the analysis of overall survival was $31^{\text {st }}$ April, 2018 corresponding to 6 months of follow-up for the last patient enrolled in the study.

\section{Patient criteria:}

- Adult $\geq 18$ years of both genders.

- ECOG performance status 0-2 at the start of treatment.
- Operable patient subjected to radical cystectomy.

- Physically fit for adjuvant chemotherapy.

- Hemoglobin $>9 \mathrm{~g} / \mathrm{dL}$

- $\mathrm{WBC} \geq 3000 / \times 10^{9} / \mathrm{L} ; \mathrm{ANC}>1500 / \times$ $10^{9} / \mathrm{L}$

- Platelet count $\geq 100,000 / \times 10^{9} / \mathrm{L}$

- Creatinine $\leq 1.2 \mathrm{mg} / \mathrm{dl}$

- Creatinine clearance $>50 \mathrm{ml} /$ minute

- Bilirubin $\leq 1$ times of upper limit of normal (ULN)

- AST, ALT $<2$ times of upper limit of normal (ULN)

- Treatment begins within 3 months after surgery.

Disease criteria: All patients had one or more of the following risk factors:

- Histopathological proven invasive bladder carcinoma p T2, T3, T4a, N0-N3.

- Involvement of one or more pelvic lymph node.

- Histopathological grade 3.

\section{Exclusion criteria:}

- Evidence of hydronephrosis.

- Uncontrolled systemic disease.

- Pregnancy.

- Prior chemotherapy treatment.

- Concurrent drugs that have potential nephrotoxicity or ototoxicity.

- Other malignancy except adequately treated basal cell carcinoma of the skin.

\section{METHODS}

I. Full history and thorough physical examination; including body surface area and performance status according to $\mathrm{WHO}$ scale.

\section{Investigations:}

Routine laboratory studies: (Not more than 4 weeks prior to study entry):

- CBC, Alkaline phosphatase, ALT, AST, bilirubin, BUN, creatinine, and calculated creatinine clearance levels

- Urine analysis and culture \& sensitivity,

Radiologic evaluation: (Not more than 6 weeks prior to study entry):

- Chest x-ray,

- Abdominal and pelvic CT scans or MRI

- Echocardiography.

- Isotopic bone scan should be performed only in patients who complain of (A) bone pain, or who have (B) an elevated serum calcium 
level or (C) an elevated serum alkaline phosphatase level.

○ IVP if indicated.

\section{Protocol Design:}

All patients were subjected to the following treatment protocol:

1. Radical cystectomy and pelvic lymphadenectomy.

2. Received four cycles of adjuvant chemotherapy cisplatin $70 \mathrm{mg} / \mathrm{m}^{2} \quad \mathrm{D} 1$, gemcitabine $1000 \mathrm{mg} / \mathrm{m}^{2} \mathrm{D} 1,8$, every three weeks.

\section{The Chemotherapy:}

The adjuvant chemotherapy

○ Cisplatinum $70 \mathrm{mg} / \mathrm{m}^{2}$ day 1 .

○ Gemcitabine $1000 \mathrm{mg} / \mathrm{m}^{2}$ days 1,8 .

\section{Administration (intravenous infusion)}

Day 1 of the adjuvant chemotherapy:

Pretreatment Hydration and Medical Preparation:

- The Patient was given one and half liters of normal saline $+20 \mathrm{ml} \mathrm{Eq}$ of potassium chloride $+1 \mathrm{gm}$ of magnesium sulphate followed by $200 \mathrm{ml}$ of mannitol $20 \%$ over 20 mints.

- Dexamethasone $16 \mathrm{mg}$ and ondansetron 16 $\mathrm{mg}$ or granisetrone $3 \mathrm{mg}$ in $100 \mathrm{ml}$ saline given as an intravenous infusion over 15 min, 30 min before cisplatin administration.
- Apripatent (EMEND) $120 \mathrm{mg}$ one hour before cisplatinum on day one, and $80 \mathrm{mg}$ on day two and three.

\section{Chemotherapy Administration:}

○ Cisplatin $70 \mathrm{mg} / \mathrm{m}^{2}$ was given in 500 $\mathrm{ml}$ of normal saline over 2 hours.

o Gemcitabine $1000 \mathrm{mg} / \mathrm{m}^{2}$ in $250 \mathrm{ml}$ normal saline over 30 minutes.

\section{Post treatment hydration:}

- One liter of normal saline $+1 \mathrm{gm}$ magnesium sulphate $+20 \mathrm{ml} \mathrm{Eq}$ of potassium chloride over 2 hours

- Patients were instructed to drink ample fluids with careful monitoring of diuresis $(500 \mathrm{ml}$ of urine output at minimum is required within the first 6 hours on day one).

\section{Day 8 of adjuvant chemotherapy}

- Dexamethasone $8 \mathrm{mg}$ and ondansetron $8 \mathrm{mg}$ or granisetrone $3 \mathrm{mg}$ in $100 \mathrm{ml}$ saline as an intravenous infusion over $15 \mathrm{~min}$.

o Gemcitabine $1000 \mathrm{mg} / \mathrm{m} 2$ in $250 \mathrm{ml}$ normal saline over 30 minute's infusion.

\section{Dose Modifications:}

\section{Dose modification for Cisplatin:}

Table (1): Modifications of Cisplatin according to Creatinine clearance

\begin{tabular}{|c|c|c|c|}
\hline Creatine clearance (CRCL) & $\mathbf{N}$ & $\mathbf{\%}$ & Dose Reduction \\
\hline$(30-44) \mathrm{ML} / \mathrm{h}$ & 1 & 2.38 & $50 \%$ Dose reduction \\
\hline$(45-60) \mathrm{ML} / \mathrm{h}$ & 8 & 19.05 & $25 \%$ Dose reduction \\
\hline$>60 \mathrm{ML} / \mathrm{h}$ & 33 & 78.57 & Nill \\
\hline
\end{tabular}

Table (2): Modifications of Cisplatin for myelosuppression

\begin{tabular}{|c|c|c|c|c|}
\hline \multicolumn{5}{|c|}{ Calculated dose \% } \\
\hline ANC $\left(/ \times 10^{9} / \mathrm{L}\right)$ (Absolute neutrophils count) & \multicolumn{4}{c|}{ Platelet count } \\
\hline & $>150.000$ & $\mathbf{1 0 0 - 1 4 9 . 0 0 0}$ & $\mathbf{7 5 - 9 9 . 0 0 0}$ & $<75.000$ \\
\hline$\geq 1.4$ & $\mathbf{1 0 0}$ & $\mathbf{1 0 0}$ & $\mathbf{1 0 0}$ & $\mathbf{7 5}$ \\
\hline $\mathbf{1 . 0}-<\mathbf{1 . 4}$ & $\mathbf{1 0 0}$ & $\mathbf{7 5}$ & $\mathbf{7 5}$ & $\mathbf{7 5}$ \\
\hline$<\mathbf{1 . 0}$ & $\mathbf{0}$ & $\mathbf{0}$ & $\mathbf{0}$ & $\mathbf{0}$ \\
\hline
\end{tabular}

II. Dose modification for Gemcitabine:

Table (3): Modifications of Gemcitabine for myelosuppresion

\begin{tabular}{|c|c|c|c|}
\hline ANC $\left(\times 10^{9} / \mathrm{L}\right)($ Absolute neutrophils count) & & Platelet $(\times 109 / \mathrm{L})$ & Percent of full dose \\
\hline$\geq 1.0$ & And & $\geq 75$ & 100 \\
\hline 0.5 to 0.99 & OR & 50 to 74 & 50 \\
\hline$<0.5$ & OR & $<50$ & Hold \\
\hline
\end{tabular}

III. Assessment schedule:

During adjuvant chemotherapy:

- Physical examination each visit.

- $\quad$ BBC, urea and creatinine before each cisplatinum administration.

- $\quad$ CBC before each Gemcitabine administration.

- Toxicity assessment was done each visit according to WHO toxicity. 
IV. Follow up:

- CBC, kidney function and liver function tests, done every two months in the first year, three months in second year and every six months thereafter.

- Chest X-ray and CT abdomen and pelvis every six months in the first two years, and every 12 months thereafter.

\section{RESULTS}

Note on Results:

1. For each table or graph, your data should be first presented collectively as a text and then presented in detail as tables or graphs.

Table (4): Patient's characteristics of study group patients

\begin{tabular}{|c|c|c|}
\hline \multicolumn{3}{|c|}{ Epidemiology } \\
\hline \multicolumn{3}{|l|}{ Age } \\
\hline Range & \multicolumn{2}{|c|}{$41-70$} \\
\hline Mean \pm SD & \multicolumn{2}{|c|}{$59.833 \pm 7.197$} \\
\hline Sex & $\mathbf{N}$ & $\%$ \\
\hline Male & 36 & 85.71 \\
\hline Female & 6 & 14.29 \\
\hline \multicolumn{3}{|l|}{ Occupation } \\
\hline Worker & 15 & 35.71 \\
\hline Farmer & 21 & 50.00 \\
\hline Driver & 2 & 4.76 \\
\hline Employee & 1 & 2.38 \\
\hline Housewife & 3 & 7.14 \\
\hline \multicolumn{3}{|l|}{ Residence } \\
\hline Upper Egypt & 14 & 33.33 \\
\hline Lower Egypt & 11 & 26.19 \\
\hline Greater Cairo & 17 & 40.48 \\
\hline \multicolumn{3}{|l|}{ Residence } \\
\hline Urban & 20 & 47.62 \\
\hline Rural & 22 & 52.38 \\
\hline \multicolumn{3}{|l|}{ Special habit } \\
\hline Non-Smoker & 9 & 21.43 \\
\hline Smoker & 33 & 78.57 \\
\hline \multicolumn{3}{|l|}{ F.H. } \\
\hline No & 42 & 100.00 \\
\hline Yes & 0 & 0.00 \\
\hline \multicolumn{3}{|l|}{ Comorbidities } \\
\hline \multicolumn{3}{|c|}{ Bilharzial Cystities } \\
\hline Negative & 29 & 69.00 \\
\hline Positive & 13 & 31.00 \\
\hline \multicolumn{3}{|l|}{$\mathrm{HCV}$} \\
\hline Negative & 28 & 66.67 \\
\hline Positive & 14 & 33.33 \\
\hline \multicolumn{3}{|l|}{ HBV } \\
\hline Negative & 42 & 100.00 \\
\hline Positive & 0 & 0.00 \\
\hline \multicolumn{3}{|l|}{ DM } \\
\hline Negative & 33 & 78.57 \\
\hline Positive & 9 & 21.43 \\
\hline \multicolumn{3}{|l|}{ HTN } \\
\hline Negative & 35 & 83.33 \\
\hline Positive & 7 & 16.67 \\
\hline \multicolumn{3}{|c|}{ Performance status (ECOG) } \\
\hline ECOG 0 & 15 & 35.71 \\
\hline ECOG 1 & 22 & 52.38 \\
\hline ECOG 2 & 5 & 11.90 \\
\hline
\end{tabular}


Table (5): Histopathologically assessment of the eligible 42 patients

\begin{tabular}{|c|c|c|}
\hline \multicolumn{3}{|c|}{ Pathological criteria: } \\
\hline & $\mathbf{N}$ & $\%$ \\
\hline T2a & 2 & 4.76 \\
\hline T2b & 6 & 14.29 \\
\hline T3a & 11 & 26.19 \\
\hline T3b & 9 & 21.43 \\
\hline T4a & 11 & 26.19 \\
\hline T4b & 3 & 7.14 \\
\hline \multicolumn{3}{|l|}{ Histopathology } \\
\hline TCC & 35 & 83.33 \\
\hline SCC & 7 & 16.67 \\
\hline \multicolumn{3}{|c|}{ Squamous differentiation } \\
\hline No & 32 & 76.19 \\
\hline Yes & 10 & 23.81 \\
\hline \multicolumn{3}{|l|}{ Grade } \\
\hline Grade II & 13 & 30.95 \\
\hline Grade III & 29 & 69.05 \\
\hline \multicolumn{3}{|l|}{ Bilharzial cystitis } \\
\hline No & 29 & 69.05 \\
\hline Yes & 13 & 30.95 \\
\hline \multicolumn{3}{|l|}{ Lymph node Total } \\
\hline Range & 1 & 29 \\
\hline Mean \pm SD & 11.048 & 7.116 \\
\hline \multicolumn{3}{|l|}{ Lymph node Status } \\
\hline No & 31 & 73.81 \\
\hline Yes & 11 & 26.19 \\
\hline Extra nodal spread & $\mathbf{N}$ & $\%$ \\
\hline No & 41 & 97.62 \\
\hline Yes & 1 & 2.38 \\
\hline \multicolumn{3}{|c|}{ Lymphovascular invasion } \\
\hline No & 35 & 83.33 \\
\hline Yes & 7 & 16.67 \\
\hline \multicolumn{3}{|l|}{ Pathological Stages } \\
\hline Stage 2 & 8 & 19.05 \\
\hline Stage 3 & 22 & 52.38 \\
\hline Stage 4a & 12 & 28.57 \\
\hline
\end{tabular}

Table (6): Relatives of dose intensities

\begin{tabular}{|c|c|c|c|}
\hline \multicolumn{4}{|c|}{ Relative dose intensities \% } \\
\hline Range & 55 & - & 100 \\
\hline Mean \pm SD & 91.517 & \pm & 10.942 \\
\hline
\end{tabular}

Table (7): Hematological toxicity

\begin{tabular}{|c|c|c|}
\hline \multicolumn{3}{|c|}{ Hematological toxicity: } \\
\hline & $\mathbf{N}$ & $\%$ \\
\hline \multicolumn{3}{|c|}{ HB } \\
\hline Grade 0 & 4 & 9.52 \\
\hline Grade I & 25 & 59.52 \\
\hline Grade II & 9 & 21.43 \\
\hline Grade III & 4 & 9.52 \\
\hline \multicolumn{3}{|c|}{ WBC } \\
\hline Grade 0 & 10 & 23.81 \\
\hline Grade I & 16 & 38.10 \\
\hline Grade II & 8 & 19.05 \\
\hline Grade III & 7 & 16.67 \\
\hline Grade IV & 1 & 2.38 \\
\hline \multicolumn{3}{|c|}{ PLT } \\
\hline Grade 0 & 33 & 78.57 \\
\hline Grade I & 6 & 14.29 \\
\hline Grade II & 2 & 4.76 \\
\hline Grade III & 1 & 2.38 \\
\hline
\end{tabular}


Adjuvant Chemotherapy Treatment after Radical Cystectomy in Patients...

Table (8): Gastrointestinal toxicity

\begin{tabular}{|c|c|c|}
\hline \multicolumn{3}{|c|}{ GIT TOXICITY: } \\
\hline \multicolumn{3}{|c|}{ ANOREXIA \& WEIGHT LOSS } \\
\hline Grade 0 & 2 & 4.76 \\
\hline Grade I & 28 & 66.67 \\
\hline Grade II & 11 & 26.19 \\
\hline Grade III & 1 & 2.38 \\
\hline \multicolumn{3}{|c|}{ NAUSEA } \\
\hline Grade 0 & 3 & 7.14 \\
\hline Grade I & 12 & 28.57 \\
\hline Grade II & 15 & 35.71 \\
\hline Grade III & 12 & 28.57 \\
\hline \multicolumn{3}{|c|}{ VOMITING } \\
\hline Grade 0 & 6 & 14.29 \\
\hline Grade I & 19 & 45.24 \\
\hline Grade II & 13 & 30.95 \\
\hline Grade III & 2 & 4.76 \\
\hline Grade IV & 2 & 4.76 \\
\hline \multicolumn{3}{|c|}{ DIARRHOEA } \\
\hline Grade 0 & 10 & 23.81 \\
\hline Grade I & 25 & 59.52 \\
\hline Grade II & 3 & 7.14 \\
\hline Grade III & 4 & 9.52 \\
\hline \multicolumn{3}{|c|}{ MUCOSITIS } \\
\hline Grade 0 & 34 & 80.95 \\
\hline Grade I & 6 & 14.29 \\
\hline Grade II & 2 & 4.76 \\
\hline
\end{tabular}

Table (9): Renal Toxicities

\begin{tabular}{|c|c|c|}
\hline \multicolumn{3}{|c|}{ RENAL TOXICITY: } \\
\hline & $\mathbf{N}$ & $\%$ \\
\hline \multicolumn{3}{|c|}{ S. Cr. } \\
\hline Grade 0 & 29 & 69.05 \\
\hline Grade I & 9 & 21.43 \\
\hline Grade II & 2 & 4.76 \\
\hline Grade III & 2 & 4.76 \\
\hline \multicolumn{3}{|c|}{ PROT. } \\
\hline Grade 0 & 31 & 73.81 \\
\hline Grade I & 10 & 23.81 \\
\hline Grade II & 1 & 2.38 \\
\hline \multicolumn{3}{|c|}{ HAEMAT. } \\
\hline Grade 0 & 33 & 78.57 \\
\hline Grade I & 8 & 19.05 \\
\hline Grade II & 1 & 2.38 \\
\hline
\end{tabular}

Survival Function

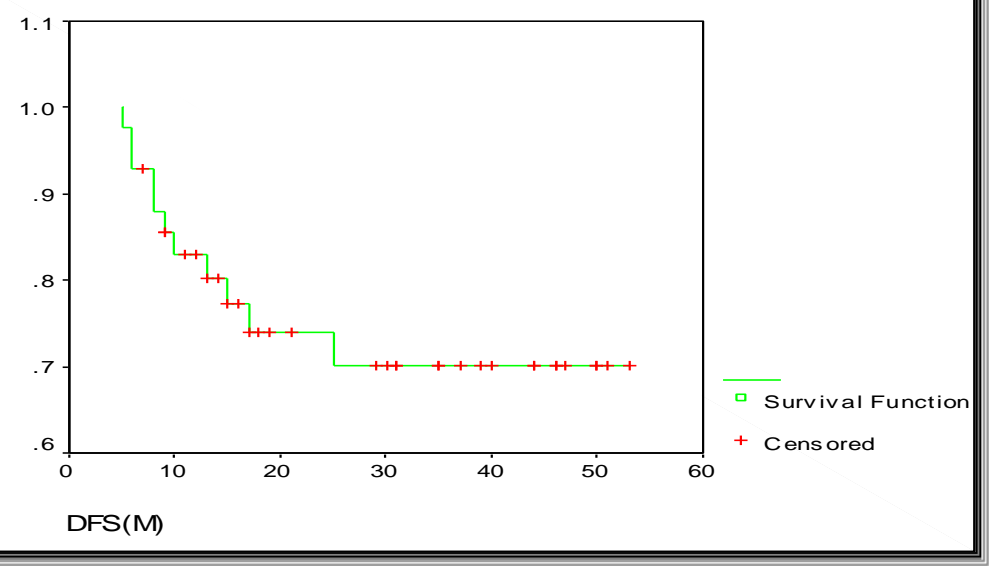

Fig. (1): Curve for disease free survival. 
Table (10): Relation between DFS and different factors of the studied group

\begin{tabular}{|c|c|c|c|c|c|c|c|}
\hline & & \multicolumn{4}{|c|}{ DFS (M) } & \multicolumn{2}{|c|}{ T-Test or ANOVA } \\
\hline & & $\mathbf{N}$ & Mean & \pm & SD & $\mathbf{T}$ or $\mathbf{F}$ & P-value \\
\hline \multirow{5}{*}{ Occupation } & Worker & 15 & 17.867 & \pm & 13.384 & \multirow{5}{*}{2.172} & \multirow{5}{*}{0.091} \\
\hline & Farmer & 21 & 26.714 & \pm & 14.585 & & \\
\hline & Driver & 2 & 42.000 & \pm & 7.071 & & \\
\hline & Employment & 1 & 17.000 & \pm & 0.000 & & \\
\hline & Housewife & 3 & 37.000 & \pm & 25.159 & & \\
\hline \multirow{3}{*}{ Residence 1} & Upper Egypt & 14 & 22.643 & \pm & 15.998 & \multirow{3}{*}{0.385} & \multirow{3}{*}{0.683} \\
\hline & Lower Egypt & 11 & 23.545 & \pm & 14.264 & & \\
\hline & Greater Cairo & 17 & 27.353 & \pm & 16.621 & & \\
\hline \multirow{2}{*}{ Residence 2} & Urban & 20 & 23.600 & \pm & 16.753 & \multirow{2}{*}{-0.465} & \multirow{2}{*}{0.644} \\
\hline & Rural & 22 & 25.864 & \pm & 14.782 & & \\
\hline \multirow{2}{*}{ Special Habit } & Non-Smoker & 9 & 29.333 & \pm & 19.455 & \multirow{2}{*}{0.986} & \multirow{2}{*}{0.330} \\
\hline & Smoker & 33 & 23.545 & \pm & 14.481 & & \\
\hline \multirow{2}{*}{ Bilharzial Cystities } & Negative & 29 & 24.172 & \pm & 15.229 & \multirow{2}{*}{-0.377} & \multirow{2}{*}{0.708} \\
\hline & Positive & 13 & 26.154 & \pm & 16.945 & & \\
\hline \multirow{2}{*}{ Surgical Types } & Non-Orthotopic & 20 & 21.750 & \pm & 13.879 & \multirow{2}{*}{-1.209} & \multirow{2}{*}{0.234} \\
\hline & Orthotopic (illeal pouch) & 22 & 27.545 & \pm & 16.852 & & \\
\hline \multirow{3}{*}{ Performance Status (ECOG) } & ECOG 0 & 15 & 27.333 & \pm & 15.523 & \multirow{3}{*}{0.370} & \multirow{3}{*}{0.693} \\
\hline & ECOG 1 & 22 & 23.909 & \pm & 15.418 & & \\
\hline & ECOG 2 & 5 & 21.000 & \pm & 18.868 & & \\
\hline \multirow{2}{*}{ Squamous Differentiation } & No & 32 & 26.750 & \pm & 15.610 & \multirow{2}{*}{1.481} & \multirow{2}{*}{0.146} \\
\hline & Yes & 10 & 18.500 & \pm & 14.539 & & \\
\hline \multirow{2}{*}{ Grade } & Grade II & 13 & 27.385 & \pm & 15.634 & \multirow{2}{*}{0.719} & \multirow{2}{*}{0.477} \\
\hline & Grade III & 29 & 23.621 & \pm & 15.715 & & \\
\hline \multirow{2}{*}{ Lymphovascular Invasion } & No & 35 & 25.229 & \pm & 15.488 & \multirow{2}{*}{0.407} & \multirow{2}{*}{0.686} \\
\hline & Yes & 7 & 22.571 & \pm & 17.213 & & \\
\hline
\end{tabular}

$\mathrm{T}$-Independent samples t-test.

F-One-way ANOVA tests.

Table (11): Relation between DFS and different factors of the studied group

\begin{tabular}{|c|c|c|c|c|c|c|c|}
\hline \multicolumn{2}{|l|}{ DFS } & $\mathbf{N}$ & $1 \mathrm{Y}$ & $2 \mathbf{Y}$ & $3 \mathbf{Y}$ & Median (95\% CI) & P-value \\
\hline \multirow{2}{*}{ Age } & $<60$ Years & 17 & 0.765 & 0.701 & 0.701 & NA & \multirow{2}{*}{0.776} \\
\hline & $\geq 60$ Years & 25 & 0.875 & 0.757 & 0.673 & NA & \\
\hline \multirow{2}{*}{ Histopathology } & TCC & 35 & 0.824 & 0.710 & 0.659 & NA & \multirow{2}{*}{0.375} \\
\hline & SCC & 7 & 0.857 & 0.857 & 0.857 & NA & \\
\hline \multirow{6}{*}{$\begin{array}{l}\text { pathological } \\
\text { T-Stages }\end{array}$} & T2a & 2 & NA & NA & NA & NA & \multirow{6}{*}{$0.002 *$} \\
\hline & T2b & 6 & 0.833 & 0.833 & 0.833 & NA & \\
\hline & T3a & 11 & 0.900 & 0.800 & 0.800 & NA & \\
\hline & T3b & 9 & 0.889 & 0.762 & 0.571 & NA & \\
\hline & T4a & 11 & 0.818 & 0.818 & 0.818 & NA & \\
\hline & T4b & 3 & 0.333 & 0.333 & 0.333 & $8(3.2-12.8)$ & \\
\hline \multirow{3}{*}{ Pathological TN Stages } & Stage 2 & 8 & 0.875 & 0.875 & 0.875 & NA & \multirow{3}{*}{$<0.001 *$} \\
\hline & Stage 3 & 22 & 0.947 & 0.947 & 0.947 & NA & \\
\hline & Stage 4a & 12 & 0.583 & 0.292 & 0.146 & $13(4.04-21.96)$ & \\
\hline \multirow{2}{*}{ Lymph node status } & No & 31 & 0.898 & 0.898 & 0.898 & NA & \multirow{2}{*}{$<0.001 *$} \\
\hline & Yes & 11 & 0.636 & 0.318 & 0.159 & $15(6.35-23.65)$ & \\
\hline
\end{tabular}

NA: not applicable, CI: confidence interval, PS: performance status. 
Adjuvant Chemotherapy Treatment after Radical Cystectomy in Patients...

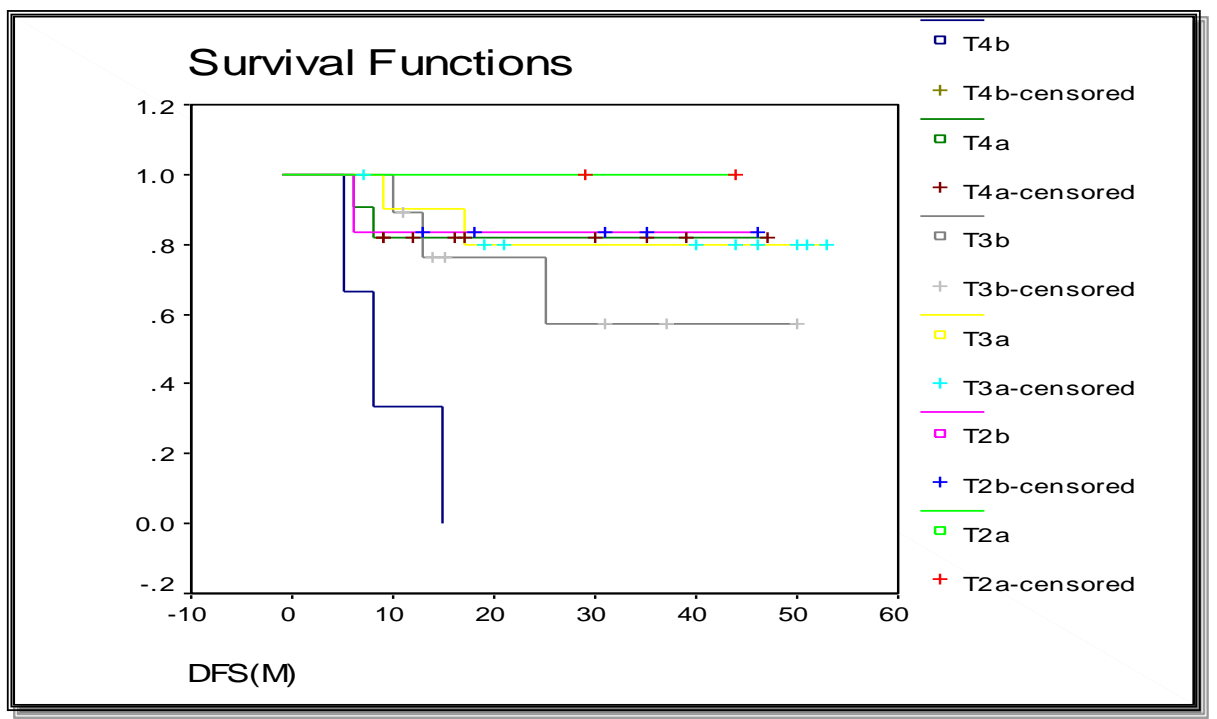

Fig. (2): Effect of pathological T-stage on DFS.

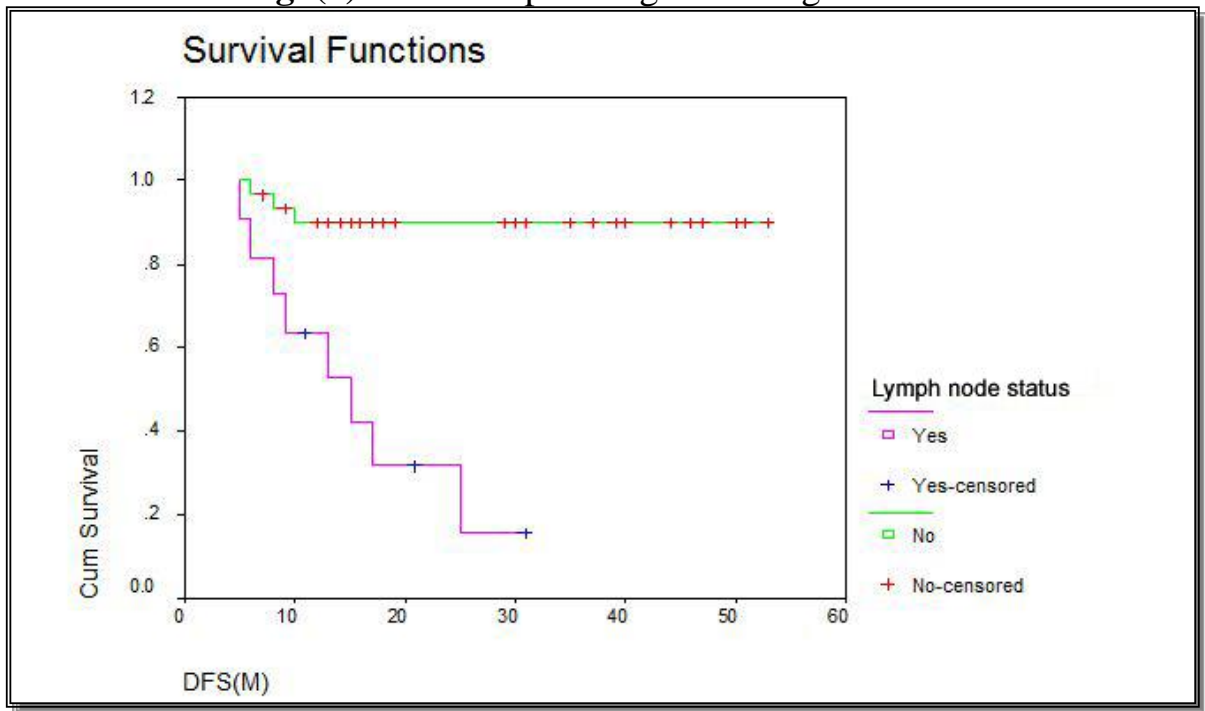

Fig. (3): Effect of positive lymph nodes on DFS.

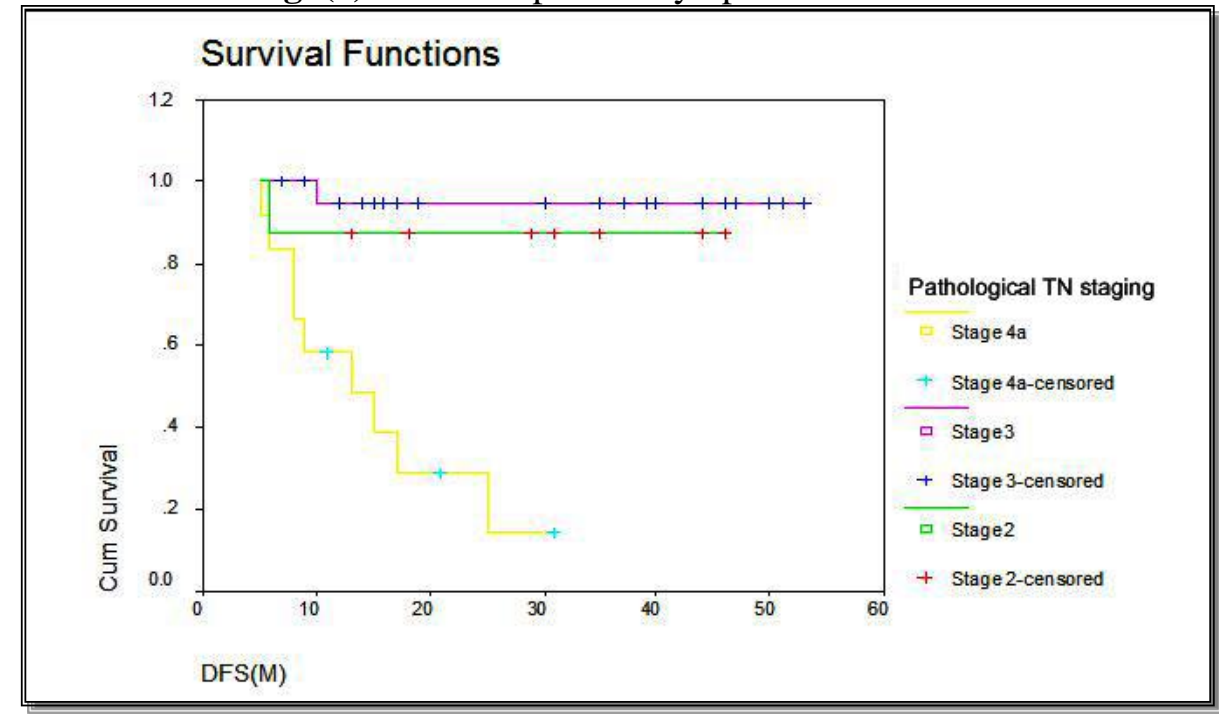

Fig. (4): Effect of pathological stages on DFS. 


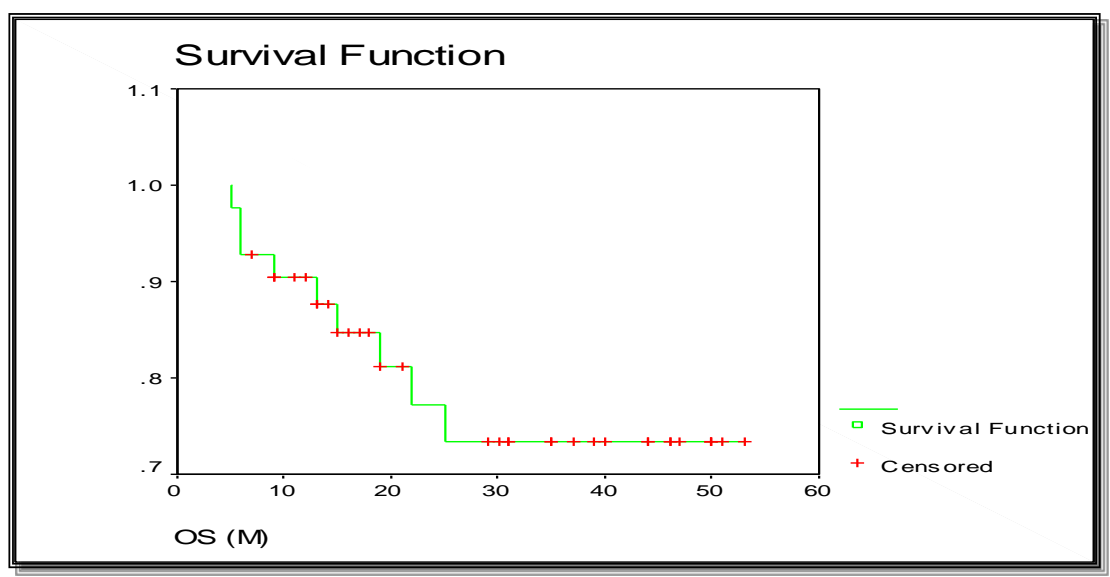

Fig. (5): Overall survival curve.

Table (12): Relation between OS and different factors of the studied group

\begin{tabular}{|c|c|c|c|c|c|c|c|}
\hline & & \multicolumn{4}{|c|}{ OS (M) } & \multicolumn{2}{|c|}{ T-Test or ANOVA } \\
\hline & & $\mathbf{N}$ & Mean & \pm & SD & $\mathbf{T}$ or $\mathbf{F}$ & P-value \\
\hline \multirow{5}{*}{ Occupation } & Worker & 15 & 18.200 & \pm & 13.181 & \multirow{5}{*}{4.909} & \multirow{5}{*}{0.343} \\
\hline & Farmer & 21 & 27.381 & \pm & 14.059 & & \\
\hline & Driver & 2 & 42.000 & \pm & 7.071 & & \\
\hline & Employment & 1 & 17.000 & \pm & 0.000 & & \\
\hline & Housewife & 3 & 51.000 & \pm & 1.732 & & \\
\hline \multirow{3}{*}{ Residence 1} & Upper Egypt & 14 & 23.286 & \pm & 15.628 & \multirow{3}{*}{0.795} & \multirow{3}{*}{0.459} \\
\hline & Lower Egypt & 11 & 24.364 & \pm & 13.735 & & \\
\hline & Greater Cairo & 17 & 29.882 & \pm & 16.605 & & \\
\hline \multirow{2}{*}{ Residence 2} & Urban & 20 & 23.850 & \pm & 16.544 & \multirow{2}{*}{-0.950} & \multirow{2}{*}{0.348} \\
\hline & Rural & 22 & 28.409 & \pm & 14.543 & & \\
\hline \multirow{2}{*}{ Special Habit } & Non-Smoker & 9 & 34.667 & \pm & 18.042 & \multirow{2}{*}{1.897} & \multirow{2}{*}{0.065} \\
\hline & Smoker & 33 & 23.939 & \pm & 14.188 & & \\
\hline \multirow{2}{*}{ Bilharzial Cystities } & Negative & 29 & 25.931 & \pm & 15.418 & \multirow{2}{*}{-0.189} & \multirow{2}{*}{0.851} \\
\hline & Positive & 13 & 26.923 & \pm & 16.317 & & \\
\hline \multirow{3}{*}{$\begin{array}{l}\text { Performance Status } \\
\text { (ECOG) }\end{array}$} & ECOG 0 & 15 & 28.133 & \pm & 14.784 & \multirow{3}{*}{0.386} & \multirow{3}{*}{0.682} \\
\hline & ECOG 1 & 22 & 26.136 & \pm & 15.713 & & \\
\hline & ECOG 2 & 5 & 21.000 & \pm & 18.868 & & \\
\hline \multirow{2}{*}{ Pathology (Sq. Diff) } & No & 32 & 27.344 & \pm & 15.120 & \multirow{2}{*}{0.823} & \multirow{2}{*}{0.415} \\
\hline & Yes & 10 & 22.700 & \pm & 17.023 & & \\
\hline \multirow{2}{*}{ Grade } & Grade II & 13 & 28.000 & \pm & 15.149 & \multirow{2}{*}{0.488} & \multirow{2}{*}{0.628} \\
\hline & Grade III & 29 & 25.448 & \pm & 15.865 & & \\
\hline \multirow{2}{*}{ Lymphovascular invasion } & No & 35 & 25.743 & \pm & 15.130 & \multirow{2}{*}{-0.458} & \multirow{2}{*}{0.649} \\
\hline & Yes & 7 & 28.714 & \pm & 18.373 & & \\
\hline
\end{tabular}

T -Independent samples t-test.

F-One-way ANOVA tests.

Table (13): Relation between OS and different factors of the studied group

\begin{tabular}{|c|c|c|c|c|c|c|c|}
\hline \multicolumn{2}{|l|}{$\mathbf{O S}$} & $\mathbf{N}$ & $1 \mathrm{Y}$ & $2 Y$ & $3 \mathrm{Y}$ & Median (95\% CI) & P-value \\
\hline \multirow{2}{*}{ Age } & $<60$ Years & 17 & 0.824 & 0.755 & 0.755 & NA & \multirow{2}{*}{0.976} \\
\hline & $>60$ Years & 25 & 0.960 & 0.770 & 0.684 & NA & \\
\hline \multirow{2}{*}{ Histopathology } & TCC & 35 & 0.914 & 0.746 & 0.696 & NA & \multirow{2}{*}{0.494} \\
\hline & SCC & 7 & 0.857 & 0.857 & 0.857 & NA & \\
\hline \multirow{6}{*}{$\begin{array}{l}\text { pathological } \\
\text { T-Stages }\end{array}$} & $\mathrm{T} 2 \mathrm{a}$ & 2 & NA & NA & NA & NA & \multirow{6}{*}{0.219} \\
\hline & $\mathrm{T} 2 \mathrm{~b}$ & 6 & 0.833 & 0.833 & 0.833 & NA & \\
\hline & T3a & 11 & 0.867 & 0.857 & 0.857 & $\overline{\mathrm{NA}}$ & \\
\hline & $\mathrm{T} 3 \mathrm{~b}$ & 9 & 0.710 & 0.700 & 0.525 & NA & \\
\hline & $\mathrm{T} 4 \mathrm{a}$ & 11 & 0.818 & 0.818 & 0.818 & NA & \\
\hline & $\mathrm{T} 4 \mathrm{~b}$ & 3 & 0.667 & 0.333 & 0.333 & $15(0-31)$ & \\
\hline \multirow{3}{*}{ Pathological TN Stages } & Stage 2 & 8 & 0.875 & 0.875 & 0.875 & NA & \multirow{3}{*}{$0.001 *$} \\
\hline & Stage 3 & 22 & 0.954 & 0.944 & 0.944 & NA & \\
\hline & Stage $4 a$ & 12 & 0.750 & 0.402 & 0.268 & $22(12.62-31.38)$ & \\
\hline \multirow{2}{*}{$\begin{array}{l}\text { Lymph node } \\
\text { status }\end{array}$} & No & 31 & 0.968 & 0.931 & 0.931 & NA & \multirow{2}{*}{$<0.001 *$} \\
\hline & Yes & 11 & 0.727 & 0.323 & 0.162 & 19(10.94-27.06) & \\
\hline
\end{tabular}

NA: not applicable, CI: confidence interval, PS: performance status. 


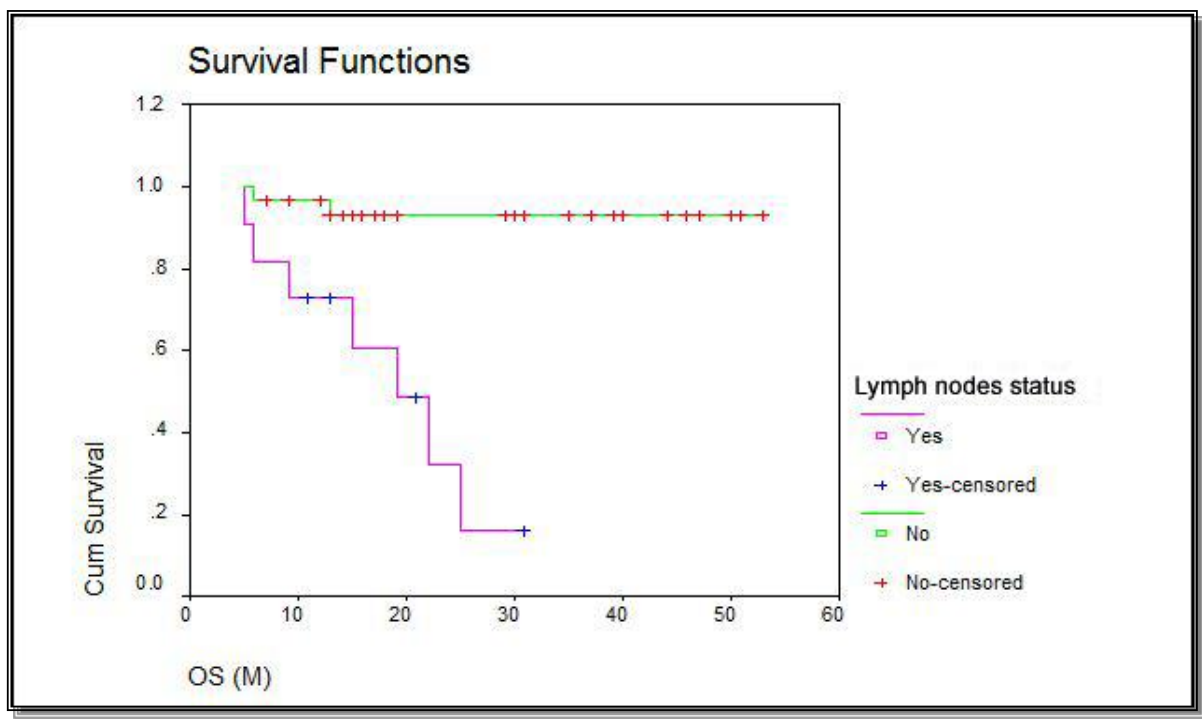

Fig. (6): Effect of positive lymph nodes on DFS.

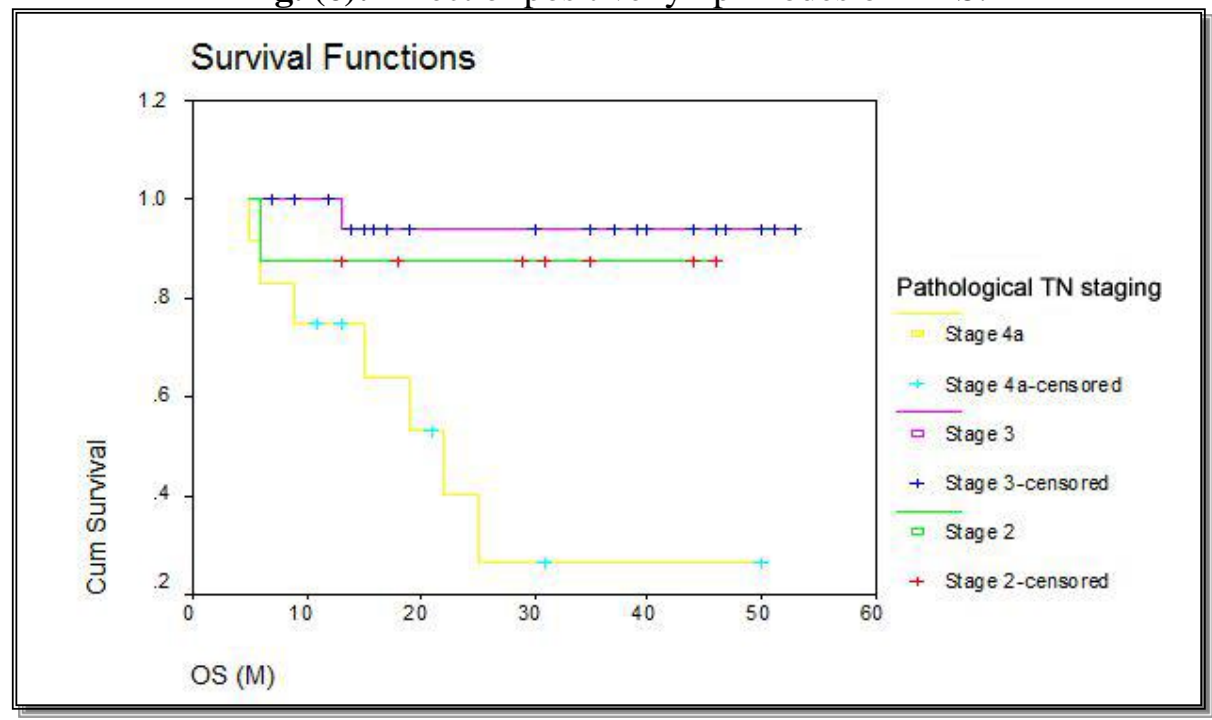

Fig. (7): Effect of pathological stages on DFS.

\section{DISCUSSION}

For patients with muscle invasive bladder cancer, cystectomy alone is associated with a 50 to 65 percent overall survival rates, which may be as high as 80 percent in patients who have pT2 disease. However, patients with locally advanced disease are at risk for worse outcomes. The five-year survival rate in patients with invasion beyond the bladder muscle is approximately 40 percent, while the survival for patients with lymph node involvement does not exceed 10 percent ${ }^{(7)}$.

Given the benefit of chemotherapy in the neoadjuvant setting and the poor prognosis of patients following surgical resection, adjuvant chemotherapy is often used in patients with high-risk bladder cancer. Although this rationale provides the justification for the use of adjuvant chemotherapy, the available data from randomized trials provide little conclusive evidence that adjuvant therapy improves survival outcomes. In addition, approximately 30 percent of patients experience complications following radical cystectomy that preclude them from receiving adjuvant chemotherapy ${ }^{(8)}$. Clinical trials are the cornerstone of evidence development in oncology, yet they are not without limitations. Clinical trials have a relatively high rate of incomplete enrollment; often not providing definitive answers to critical questions. This phenomenon more apparent in the setting of adjuvant chemotherapy for bladder cancer, with all three contemporary clinical trials addressing this question terminating prematurely because of poor accrual ${ }^{(9)}$.

A series of randomized clinical trials over the past 30 years have explored the efficacy of adjuvant chemotherapy in locally advanced 
bladder cancer. Early trials were instrumental in demonstrating feasibility but used suboptimal chemotherapy regimens, were underpowered, and/or suffered from methodological flaws, thus yielding conflicting results. Three trials Cognetti et al. ${ }^{(4)}$; Paz-Ares et al. ${ }^{(5)}$ and Sternberg et al. ${ }^{(10)}$ sought to evaluate contemporary chemotherapy regimens in the adjuvant setting in patients with locally advanced bladder cancer post cystectomy. Unfortunately, all three trials closed early because of poor accrual, collectively enrolling less than $40 \%$ of their target ${ }^{(9)}$.

The resulting evidence gap has fueled controversy regarding the role of adjuvant chemotherapy. Clinical practice guidelines offer mixed guidance, with the National Comprehensive Cancer Network guidelines supporting adjuvant chemotherapy as a category $2 \mathrm{~B}$ recommendation (i.e., based on lower-level evidence) and the European Association of Urology guidelines stating that "neither randomized trials nor a meta-analysis has provided sufficient data to support the routine use of chemotherapy ${ }^{\left({ }^{9}\right)}$.

In this study of adjuvant chemotherapy treatment by cisplatin/gemzar, after radical cystectomy in patients with muscle invasive bladder cancer, were designed to show the efficacy of adjuvant chemotherapy treatment regarding survival in comparison to its toxicity. The tested regimen was found effective with manageable acute toxicity when appropriate supportive care was employed.

In our study the mean age was $59.8( \pm 7.1)$ years. 36 patients $(85.7 \%)$ were males and 6 patients $(14.2 \%)$ were females, 33 patients $(78.5 \%)$ were smokers and 9 patients $(21.4 \%)$ non-smoker.

The performance status (PS) of patients ranged from 0-II WHO. Fifteen patients $(35.7 \%)$ were presented with performance status (PS) 0 WHO, twenty two (52.3) presented with (PS) I WHO, while 5 patients (11.9\%) with (PS) II.

The whole study group showed transitional cell carcinoma in 35 patients (83.3) and only 10 patients $(23.8 \%)$ of that patient had squamous differentiation, while squamous cell carcinoma in 7 patients (16.6\%), grade II in 13 patients $(30.9 \%)$, and grade III was found in 29 patients $(69 \%)$.

Fourteen patients $(33.2 \%)$ presented with T4 staging, while T3 staging was found in 20 patients $(47.5 \%)$, and only 8 patients (18.9\%) had T2 staging. Regional lymph node involvement was observed in 11 patients
(26.1\%), only one patient had extra nodal spread (2.3), and 7 patients had lymphovascular invasion (16.6).Total number of lymph nodes excision ranging from one to twenty nine with mean 11 lymph node excision.

Eight patients $(19 \%)$ presented with stage two, 22 patients $(52.3 \%)$ were stage III and 12 patients (28.5\%) were stage IVA disease.

An intergroup, open-label, randomized, phase 3 trial (EORTC 30994): Recruited patients from hospitals across Europe and Canada. No age limits were applied, but patients had to have a good performances status (WHO 0 or 1), adequate hematological function (white blood cell count $\geq 3.5 \times 10^{9}$ cells per $\mathrm{L}$ and platelet count $\geq 120 \times 10^{9}$ cells per L), adequate renal function (glomerular filtration rate $\geq 60 \mathrm{~mL} / \mathrm{min}$ ), and normal auditory and cardiac function. Patients with previous systemic chemotherapy or radiation to the bladder and patients regarded as unfit for cisplatin- containing combination chemotherapy or with grade 2 or worse peripheral neuropathy were ineligible. Eligible patients had histologically proven urothelial carcinoma of the bladder, pT3-pT4 disease or node positive (pN1-3) M0 disease after radical cystectomy and bilateral lymphadenectomy, with no evidence of any microscopic residual disease.

An Italian, multicenter, randomized phase III trial: Eligible patients were required to have an Eastern Cooperative Oncology Group performance status (PS) of two or less, age $\leq 75$ years, adequate bone marrow reserve and a good renal (creatinine level $\leq 1.25 \mathrm{umol} / \mathrm{l}$, measured creatinine clearance $>60 \mathrm{ml} / \mathrm{min}$ ) and liver function. A radical cystectomy with no residual disease and a minimum of 10 lymph nodes dissection was required. Randomization was required within 10 weeks after surgery. Neither prior neoadjuvant chemotherapy nor radiotherapy was allowed. One hundred and ninety-four patients with histologically proven transitional cell carcinoma of the bladder pT2 G3 (N0-2), pT3-4 (N0-2) any G or pN1-2, any Tumor (T), any $\mathrm{G}$ were considered eligible.

In our study forty two patients subjected to radical cystectomy either orthotopic type 22 patients $(52.3 \%)$ and non orthotopic type 20 patients $(47.6 \%)$, the most common complication after surgery was urinary tract infection (UTI) in all patients, while the second most common was urinary 
incontinence. these patients followed by adjuvant combination chemotherapy treatment, patients were received four cycles of cisplatin $70 \mathrm{mg} / \mathrm{m}^{2}$ day one every three weeks and gemcitabine $1 \mathrm{gm} / \mathrm{m}^{2}$ day one and day eight. Thirty Patients $(71.4 \%)$ were received adjuvant treatment less than two months from surgery, while 12 patients $(28.5 \%)$ received treatment more than two months.

In phase 3 trial (EORTC 30994), Within 90 days of cystectomy, patients were centrally randomly assigned (1:1) by minimization to either immediate adjuvant chemotherapy (four cycles of gemcitabine plus cisplatin, or high-dose methotrexate, vinblastine, doxorubicin, and cisplatin [high-dose MVAC], or MVAC) or six cycles of deferred chemotherapy at relapse, with stratification for institution, pT category, and lymph node status according to the number of nodes dissected. Overall survival was the primary endpoint; all analyses were by intention to treat. The trial was closed after recruitment of 284 of the planned 660 patients ${ }^{(\mathbf{1 0})}$.

An Italian, multicenter, randomized phase III trial: Patients were randomly allocated to control (92 patients) or to four courses of AC (102 patients). These latter patients were further randomly assigned to receive gemcitabine $1000 \mathrm{mg} / \mathrm{m} 2$ days 1,8 and 15 and cisplatin $70 \mathrm{mg} / \mathrm{m} 2$ day 2 or gemcitabine as above plus cisplatin $70 \mathrm{mg} / \mathrm{m} 2$ day 15 , every 28 days ${ }^{(4)}$.

Spanish Oncology Genitourinary Group (SOGUG) 99/01 study: Eligibility criteria included: (1) resected high-risk muscle invasive bladder carcinoma (pT3-4 and/or $\mathrm{pN}+$ ), (2) ECOG PS 0-1, (3) adequate renal function $(\mathrm{CrCl}>50 \mathrm{ml} / \mathrm{min})$, (4) $\leq 8$ weeks post-cystectomy, (5) no relevant comorbidities, and (6) signed informed consent. Eligible patients were assigned to observation or 4 courses of PGC (paclitaxel $80 \mathrm{mg} / \mathrm{m}^{2} \mathrm{~d} 1$ and 8 , gemcitabine $1000 \mathrm{mg} / \mathrm{m}^{2} \mathrm{~d} 1$ and 8 and cisplatin $70 \mathrm{mg} / \mathrm{m}^{2} \mathrm{~d} 1$ ) q21 days. The primary objective was overall survival (OS) ${ }^{(5)}$.

In this study adverse events were an important factor studied in this study as the addition of adjuvant $\mathrm{CTH}$ to radical cystectomy could affect the survival without causing significant toxicity. Eighty five percent of all patients completed the planned four cycles.

The most common grade 3 and 4 adverse events of hematological and nonhematological toxicities recorded during adjuvant chemotherapy were neutropenia
(18.8\%), grade 3 anemia (9.5\%), grade 3 thrombocytopenia $(2.3 \%)$, grade 3 nausea (28.5\%), grade 3 and 4 vomiting $(9.4 \%)$, grade 3 diarrhea $(9.4 \%)$ while grade 3 renal toxicities observed in two patients (4.7\%).

Fatigue and bony aches were also common symptoms in patients receiving adjuvant treatment. Fatigue occurred mostly in all patients. These symptoms are graded, grade 3 and 4 had occurred in (20\%) of patients, and the fact that they were life threatening, they are still an important factor affecting patients' compliance to treatment and quality of life. In multiple instances, they were the cause of treatment delay, reduction and treatment cessation.

In phase 3 trial (EORTC 30994), the toxicity adjuvant treatment had a considerably higher rate, Grade 3-4 myelosuppression was reported in 33 (26\%) of 128 patients who received treatment in the immediate chemotherapy group versus 24 (35\%) of 68 patients who received treatment in the deferred chemotherapy group, neutropenia occurred in $49(38 \%)$ versus $36(53 \%)$ patients, respectively, and thrombocytopenia in 36 (28\%) versus 26 (38\%). Two patients died due to toxicity, one in each group.

However, the toxicity was less compared to the Italian, randomized phase III trial, $62 \%$ of patients received the planned four cycles, mainly due to treatment-related toxic effects. In the B2 arm, 67\% required dose adjustment and $39 \%$ patients required an early stop treatment. In the B15 arm, a dose reduction and an early stop treatment was required for $72 \%$ and $26 \%$ patients, respectively. All the WHO hematologic and non-hematologic toxic effects observed in B2 and B15 arms. A statistically significant higher incidence of grade 3/4 thrombocytopenia was observed in B2 arm as compared with B15 arm (25.6\% versus $4.3 \%, \mathrm{P}=0.006$ ). On the contrary, patients in B15 arm experienced more grade $3 / 4$ leukopenia $(15.2 \%$ versus $9.3 \%)$ and neutropenia (34.8\% versus $21 \%$ ), not reaching statistical significance. The incidence of grade 3 and 4 nausea and vomiting was higher in B2 arm $(9.4 \%$ versus $2.2 \%, \mathrm{P}=0.31)$. No drug toxicity-related death was observed in either arm.

In (SOGUG) 99/01 study, 76\% of patients completed all 4 courses of therapy in the PGC arm. the toxicity during adjuvant treatment had a considerably higher rate of 
grade 3-4 toxicities were neutropenia $41 \%$, febrile neutropenia $8 \%$, thrombocytopenia $14 \%$, anemia $5 \%$, fatigue $14 \%$, alopecia $10 \%$, vomiting $8 \%$, renal $5 \%$. There was one toxic death (sepsis).

As regard the survival analysis, the median disease free survival rate was not reached due to a relatively short follow up period and DFS was $82.9 \%$ at 1 year, $74 \%$ at 2 years, and $70.1 \%$ at 3 years, These results are comparable to immediate treatment group in phase 3 trial (EORTC 30994) by Sternberg et al. ${ }^{(10)}$, it was $78 \%, 59 \%, 50 \%$ respectively, Median diseasefree survival was 37 months (95\% CI 1.84-7.77) in the immediate treatment group compared with 11 months $(0.63-1.49)$ in the deferred treatment group (HR 0.54, 95\% CI 0.40-0.73; p<0.0001;), 5 -year disease -free survival was $47.6 \%$ (95\% CI $38.8-55.9)$ in the immediate treatment group and $31.8 \%(24.2-39.6)$ in the deferred treatment group.

This superiority in our results at two and three years of DFS, probably due to the consistent percentage of node-negative patients included, inclusion of early pathological stages (pT2N0) and bladder cancer pathology of squamous cell carcinoma while they were not included in (EORTC 30994) trial.

Our results were also better than the results by Cognetti et al. ${ }^{(4)}$, DFS of (AC) arms was $68 \%$ at 1 year, $50 \%$ at 2 years, and $44.2 \%$ at 3 years, the trial failed to confirm any survival advantage associated with adding adjuvant treatment, The control and $\mathrm{AC}$ arms were almost comparable relative to disease-free survival: $42.3 \%$, arm $\mathrm{A}$ and $37.2 \%$, arm $\mathrm{B}(\mathrm{P}=$ 0.70, HR 1.08; 95\% CI 0.73-1.59).

In the subgroup analysis according to nodal status, the 5-year disease-free survival of the node-negative patients was $59.5 \%$ in the control arm and $57.6 \%$ in the $\mathrm{AC}$ arm $(\mathrm{P}=$ $0.97)$. In node- positive patients, 5-year diseasefree survival was $19.4 \%$ in the control group and $18.9 \%$ in the AC group $(\mathrm{P}=0.80)$.

Concerning overall survival analysis, the median overall survival in our study was not reached due to a relatively short follow up period. Overall Survival rate at 1 year was $90.4 \%$; at 2 years was $77.3 \%$ and $73.4 \%$ at 3 years. Comparably, In (EORTC 30994) trial, the immediate treatment arm had a median OS of 80.5 months (95\% CI 3.85-not reached), OS was $88 \%$ at 1 year, $75.4 \%$ at 2 years, and $64.1 \%$ at 3 years, our results seems to be equal to the EORTC 30994trial. The 5-year overall survival was $53.6 \%(95 \%$ CI $44.5-61.8)$ in the immediate treatment group and $47.7 \%$ (39.155.8 ) in the deferred treatment group with no significant difference between two groups.

Sternberg et al. ${ }^{(10)}$, The duration of survival after progression was longer in the deferred treatment group than in the immediate treatment group (HR $1 \cdot 45,95 \%$ CI $1 \cdot 02-2 \cdot 07$; $\mathrm{p}=0.037$ ). In particular, patients with local or locoregional progression in the deferred group had a median survival of 2.31 years $(95 \% \mathrm{CI}$ $0 \cdot 94-5 \cdot 14)$ after starting treatment versus $1 \cdot 11$ years (95\% CI $0.51-1.49)$ after starting treatment in those with local or locoregional progression in the immediate treatment group.

Cognetti et al. ${ }^{(4)}$, the trial failed to confirm any survival advantage associated with adding adjuvant treatment, The 5-year OS of the whole series was $48.5 \%$ (standard error $4.2 \%$ ), with no significant difference between the two arms $(\mathrm{P}=0.24): 53.7 \%$ in the control group and $43.4 \%$ in the AC arm. Our results was better than the results by Cognetti et al. ${ }^{(4)}$, OS of (AC) group was $65 \%$ at 2 years, and $54 \%$ at 3 years comparable to 77.3 at two year and 73.4 at three year.

In the subgroup analysis according to nodal status, In patients with lymph nodenegative disease, 5-year OS rates were $73.2 \%$ in the control arm and $64.5 \%$ in the $\mathrm{AC}$ arm (P $=0.65)$, in contrast to results of EORTC trial which showed survival benefit, 5-year overall survival was $79.5 \%(95 \%$ CI $63 \cdot 0-89 \cdot 2)$ in the immediate treatment group and 59.0\% (42.6$72.2)$ in the deferred treatment group (HR 0.37 , $95 \%$ CI $0 \cdot 16-0 \cdot 83 ; \mathrm{p}=0 \cdot 012$ ).

Whereas in patients with lymph node involvement, OS rates were $27.6 \%$ and $25.8 \%$ in the control and $\mathrm{AC}$ groups, respectively $(\mathrm{P}=$ 0.71). However, the results are similar to EORTC trial, with no addition of survival benefit between both arms in lymph node involvement, 5-year overall survival was $42.7 \%(32.3-52.8)$ in the immediate treatment group and $42.9 \%(32.9-52.6)$ in the deferred treatment group (HR 0.94, 0.65-1.34; $\mathrm{p}=0 \cdot 72)$.

Paz-Ares et al. ${ }^{(5)}$ reported a trial (Spanish Oncology GU Group 99/01) evaluating adjuvant paclitaxel, gemcitabine, and cisplatin (PGC) that showed a progressionfree survival benefit at five years compared to control $(\mathrm{P}<0.0001)$ after four cycles of adjuvant PGC in patients with high-risk MIBC (pT3-T4 and/or pN+). Results also showed a prolonged five-year overall survival in the PCG 
arm (60\%) compared to (53.6\%) of immediate treatment group in (EORTC) trial. However, this study was terminated early due to poor recruitment (140 enrolled out of an expected 340)

In fact, Yelfimov et al. ${ }^{(11)}$ investigated 675 patients who underwent RC for pT2-4N03. A total of 80 patients (12\%) received $\mathrm{AC}$ and were compared with the non-AC group. In this study, when controlling for age, sex, stage, and performance status in multivariate analysis, AC was associated with a $29 \%$ decrease in the risk of bladder cancer death.

Meta-analysis of nine RCTs (five previously analyzed, one updated, and three new), which included 945patients, was performed in $2013^{(3)}$. It showed $\mathbf{2 3 \%}$ relative decrease in the risk of death with AC compared with controls and $\mathbf{3 4 \%}$ relative decrease in the risk of recurrence. Although it was thought that this updated meta-analysis offered further evidence of OS and DFS benefits, there were some limitations and it is still controversial. First, individual patient's data (IPD) for this meta-analysis was not available. Second, the most recent Italian ${ }^{(4)}$ and Spanish trials ${ }^{(5)}$ had completely opposite results.

Those different results may be attributable to the different regimens used and because of patient selection bias. The Italian trial enrolled 194 patients and reported a nonsignificant OS HR of 1.29 (95\%CI, 0.84-1.99) and a non-significant DFS HR of 1.08 (95\% CI, 0.73-1.59), although mortality hazards were significantly correlated with pT stage (stage pT3 or higher) and lymph node status in a multivariate analysis. In contrast, the Spanish trial, enrolled 142 patients and demonstrated statistically significant benefits of OS and DFS, with HR of 0.38 (95\% CI, 0.22-0.65) and HR of 0.38 (95\% CI, 0.25-0.058), respectively ${ }^{(3)}$.

Overall survival benefit for adjuvant chemotherapy over the deferred chemotherapy group (HR 0.77, 95\% CI 0.65-0.91; $\mathrm{p}=0.002$ ). In particular, when restricting to the Italian, Spanish, and EORTC studies that mostly used gemcitabine plus cisplatin, severe heterogeneity was noted between the study results (heterogeneity $p=0.002$ ) and $a$ borderline significant benefit of immediate gemcitabine plus cisplatin chemotherapy was noted (HR for overall survival $0.79,95 \% \mathrm{CI}$ $0 \cdot 62-1 \cdot 00 ; \mathrm{p}=0.05)^{(3)}$.

\section{CONCLUSION}

- Despite potentially curative-intent radical cystectomy, approximately one-half of patients with deep muscle-invasive bladder cancer involving the muscularis propria (T2), perivesical tissue (T3), or pelvic structures (T4, including prostatic stroma, seminal vesicles, uterus, vagina, pelvic side wall or abdominal wall) develop metastatic disease within two years and most will succumb to their disease.

- For patients with bladder cancer who were not treated with neoadjuvant chemotherapy, we suggest not routinely administering chemotherapy following cystectomy (Grade 2C). However, for patients with high-risk (T3 or higher, pathologic node involvement) urothelial carcinomas who are candidates for cisplatin -based combination chemotherapy and are willing to accept the risk for treatmentrelated toxicities in the absence of high level of evidence, adjuvant chemotherapy is a reasonable option. If administered, we prefer to use a cisplatin-based combination.

\section{REFERENCES}

1. Stein JP, Lieskovsky G, Cote $\mathrm{R}$ et al. (2001): Radical cystectomy in treatment of invasive bladder cancer: long term results in 1054 patients. JCO., 19: 666675.

2. Xylinas E, Cha EK, Sun M et al. (2012): Risk stratification of pT1-3N0 patients after radical cystectomy for adjuvant chemotherapy counselling. Br J Cancer, 107: 1826-1832.

3. Leow JJ, Martin-Doyle W, Rajagopal PS et al. (2014): Adjuvant chemotherapy for invasive bladder cancer: a 2013 updated systematic review and metaanalysis of randomized trials. Eur Urol., 66: 42-54.

4. Cognetti F, Ruggeri EM, Felici A et al. (2012): Adjuvant chemotherapy with cisplatin and gemcitabine versus chemotherapy at relapse in patients with muscle-invasive bladder cancer submitted to radical cystectomy: An Italian, multicenter, randomized phase III trial. Ann Oncol., 23: 695-700.

5. Paz-Ares LG, Solsona E, Esteban E et al. (2010): Randomized phase III trial comparing adjuvant paclitaxel/gemcitabine/cisplatin (PGC) to observation in patients with resected 
invasive bladder cancer: Results of the Spanish Oncology Genitourinary Group (SOGUG) 99/01 study. J Clin Oncol., 28: 346-352.

6. Gallagher DJ, Milowsky MI, Iasonos A et al. (2009): Sequential adjuvant chemotherapy after surgical resection of high-risk urothelial carcinoma. Cancer, 115: 5193.

7. Sternberg CN, Donat SM, Bellmunt J et al. (2007): Chemotherapy for bladder cancer: treatment guidelines for neoadjuvant chemotherapy, bladder preservation, adjuvant chemotherapy, and metastatic cancer. Urology, 69: 62.
8. Donat SM, Shabsigh A, Savage C et al. (2009): Potential impact of postoperative early complications on the timing of adjuvant chemotherapy in patients undergoing radical cystectomy: a highvolume tertiary cancer center experience. Eur Urol., 55: 177.

9. Matthew DG, Kristian DS, Erin M et al. (2016): Effectiveness of Adjuvant Chemotherapy for Locally Advanced Bladder Cancer. J Clin Oncol., 34: 825832.

10. Sternberg CN, Skoneczna I, Kerst JM et al. (2015): Immediate versus deferred chemotherapy after radical cystectomy in patients with pT3-pT4orN+M0 urothelial carcinoma of the bladder (EORTC 30994): An intergroup, open-label, randomised phase 3 trial. Lancet Oncol., 16: 76-86.

11. Yelfimov DA, Frank I, Boorjian SA et al. (2014): Adjuvant chemotherapy is associated with decreased mortality after radical cystectomy for locally advanced bladder cancer. World J Urol., 32: 1463-1468. 